\title{
COEFFICIENTS THERMIQUES ET ECOLOGIE DE QUELQUES PLANAIRES D'EAU DOUCE 4. - LA REPRODUCTION DE DUGESIA GONOCEPHALA
}

par E. Pattee.

Dans la nature, Dugesia gonocephala (Dugès) se présente comme le troisième Triclade rhéophile, celui dont le domaine, succédant à ceux de Crenobia alpina, puis de Polycelis felina, se situe le plus en aval dans les ruisseaux de montagne. La succession des trois espèces est d'une observation courante, non seulement dans les régions alpines, mais aussi dans d'autres massifs moins élevés d'Europe Occidentale [Steinmann 1907, Van Oye 1941] ou Centrale [FLösSNER 1963].

De ce fait, nombreux sont les auteurs qui, telle BL.̈̈SING [1953], qualifient $D$. gonocephala d'eurytherme. BEcker $[1960]$ donne les températures de 2 et $27^{\circ} \mathrm{C}$ comme limites de son habitat au Wurtemberg, Beauchamp et Ullyot [1932] celles de 8 et 20$23^{\circ}$, Stankovic [1935] celles de 8 et $27^{\circ}$ dans les Balkans. Précisant davantage, Alause [1968] la voit occuper, dans le Languedoc méditerranéen, une zone limitée en amont par la température de $15^{\circ}$, en aval par celles de $9^{\circ}$ en hiver et $21^{\circ}$ en été, cette dernière valeur présentant seule les caractères d'une limite nettement marquée.

Du point de vue expérimental, l'article $\mathrm{n}^{\circ} 2$ de la présente série [Pattee 1968] traitait de la tolérance thermique de $D$. gonocephala, tolérance qui se révélait parfaite jusque vers $22^{\circ} \mathrm{C}$, c'est à dire à des températures susceptibles d'éliminer $C$. alpina et $\boldsymbol{P}$. felina en une dizaine de jours. A $27,5^{\circ}$ le temps moyen de survie de $D$. gonocephala était encore de 2 jours. L'étagement habituel des trois espćces peut donc être attribué, en partie, à leur tolérance thermique: des populations de chacune d'entre elles, entraînées au-delà de leur limite aval dans le ruisseau, auraient toutes chances d'être anéanties par le réchauffement estival.

Mais cet effet létal de la chaleur ne permet pas d'expliquer pourquoi chacune n'occupe pas la zone entière correspondant à ses possibilités de survie. Avec Steinmann [1907] il convient, en particulier, d'attirer l'attention sur la limite amont des différentes 
zones, là où la température semble devenir «trop basse 》 pour l'espèce considérée, bien qu'aucun effet létal ne s'y manifeste.

Or la compétition que se livrent les trois Planaires rhéophiles a été reconnue dès 1895 par VorgT, contestée ensuite dans diverses stations par d'autre auteurs, et démontrée de façon particulièrement probante par BEaUchamp et Ullyot [1932] en Europe Centrale. Dans la région étudiée par ces derniers, lorsque $D$. gonocephala est seule dans un ruisseau, elle occupe nous l'avons vu, une zone dont la température est comprise entre 8 et $22^{\circ}$ environ. En présence de $\boldsymbol{P}$. felina, elle n'occupe plus que la zone chaude, de température supérieure à $15^{\circ}$. Elle semble donc chassée de la partie la plus froide de son habitat. La valeur de $15^{\circ}$ marque la limite eníre les deux espèces.

Une publication précédente [PatTeE 1969a] a montré que l'issue de la compétition entre $C$. alpina et $P$. felina est déterminée, à chaque température, par la capacité reproductive de l'une et de l'autre : à $5^{\circ}$, celle de $C$. alpina l'emporte, à $10^{\circ}$ celle de $P$. felina. Le but de la présente publication est de montrer dans quelle mesure cette notion peut être étendue à $D$. gonocephala.

La littérature fournit peu de renseignements à cet égard. $L^{\prime}$ " optimum » de l'animal se situerait, selon ABELoos [1929] et DaHM [1958] au voisinage de 12 à $15^{\circ}$. Les individus observés dans la nature par BECKer [1960] atteignaient leur maturité sexuelle à des températures comprises entre 7 et $13^{\circ}$ environ, ceux observés par Steinmann [1907] entre 13 et $17^{\circ}$. Ces valeurs sont, en général, supérieures à celles que l'on note pour les deux autres espèces.

\section{1. - Matériel}

Les individus qui ont servi de parents à tout l'élevage ont été récoltés dans un petit ruisseau de plaine, portant le nom de ruisseau des Vosges (bien qu'il n'ait aucun rapport avec le massif montagneux du même nom). Il s'agit d'un affluent de rive gauche de la Saône, coulant à une dizaine de kilomètres au nord de la ville de Lyon.

Ces animaux ont une reproduction uniquement sexuée, un appareil génital dépourvu d'organes musculo-glandulaires, et appartiennent donc, selon Benazzi [1955, 1960] à l'espèce Dugesia gonocephala (Dugès) sensu stricto qui habite le continent européen. Les cocons déposés sont de grande taille et pédonculés, de couleur initialement jaune, virant en quelques heures au rouge puis au brun-noir. Une certaine proportion d'entre eux, variable selon les conditions, se montre stérile. Parmi ces cocons stériles, le plus grand nombre offre le même aspect que les cocons fertiles, mais 
ne renferme, à terme, qu'une substance blanche, pâteuse et inorganisée. D'autres cocons stériles, qui seront dénommés "coques vides» se présentent dès la ponte avec leur seule enveloppe protectrice, l'intérieur ne contenant que de l'eau qui leur confère un aspect clair et translucide. Bien que j'aie généralement trouvé une fente dans la paroi de ces cocons, il ne m'est pas possible de dire si l'animal en sécrète uniquement la coque, s'il résorbe l'intérieur avant la ponte ou s'il s'en nourrit en introduisant son pharynx à travers l'enveloppe encore tendre, ce qui représenterait une forme de cannibalisme. Comme nous le verrons, à certaines températures élevées, le nombre de ces coques vides devient appréciable.

\section{2. - Méthodes d'élevage et influence de l'agitation de l'eau}

Dans un but d'homogénéité, les méthodes adoptées sont les mêmes que celles utilisées précédemment [PATtEl 1969a], à ceci près que $D$. gonocephala atteignant des dimensions bien supérieures à cel'es de Crenobia alpina et Polycelis felina, les divers lots sont constitués de 5 individus par bol au lieu de 10 .

Les cycles de ponte entrecoupés de phases de sénilité [AвELoos 1929], assez évidents sur certains individus isolés, se trouvent masqués, dans les lots de 5 , par le déphasage entre les différents membres du groupe. Comme dans les élevages de ce dernier auteur, les pontes ont lieu toute l'année lorsque la température est constante.

Je ne suis pas parvenu à mettre au point une méthode susceptible de permettre l'élevage régulier de ces diverses Planaires en eau agitée pendant la durée de plusieurs années nécessaire à l'étude de leur cycle vital. Aussi, pour les unes comme pour les autres, la plupart des mesures ont-elles été effectuées en eau stagnante. Il s'agit cependant d'espèces rhéophiles. D. gonocephala l'est assez strictement dans la nature [DAtnM 1958]; elle recherche, aul laboratoire, des courants d'une relative importance [PATteE et Bournaud 1970].

Il m'a donc semblé utile de contrôler la méthode employée et de voir dans quelles conditions les résultats acquis pouvaient être appliqués aux milieux naturels. Parallèlement aux élevages en eau stagnante, j'ai maintenu, à 15 et à $20^{\circ}$, quelques lots de $D$. gonocephala en eau agitée et oxygénée par insufflation d'air. L'étude comparative a porté sur 3 paramètres de la reproduction : le nombre de cocons produits, parmi ceux-ci la proportion de cocons fertiles, enfin le nombre de jeunes libéré par chaque cocon fertile. Les valeurs obtenues sont inscrites au tableau I. 


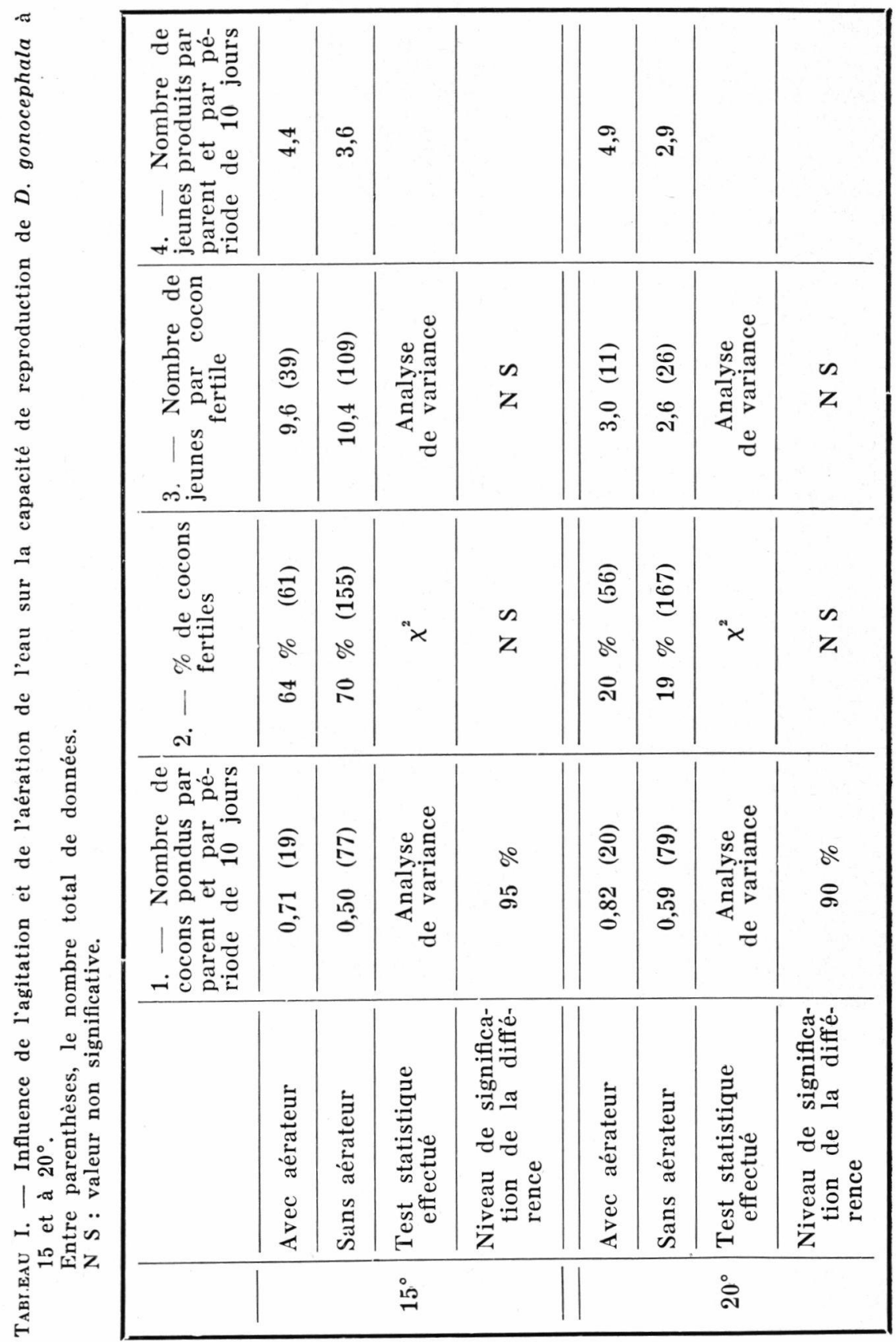


A $15^{\circ}$, l'agitation de l'eau a une influence significative sur la ponte des parents : ceux-ci déposent davantage de cocons en eau agitée ( $1^{\text {re }}$ colonne). Mais la fertilité n'est pas modifiée : il y a, des deux côtés, pratiquement la même proportion de cocons fertiles et de cocons stériles ( $2^{\mathrm{e}}$ colonne). Il y a aussi pratiquement le même nombre de jeunes par cocon ( $3^{\mathrm{e}}$ colonne). Les valeurs de la $4^{\circ}$ colonne, obtenues par calcul à partir des trois précédentes décrivent le phénomène globalement : davantage de descendants sont produits en milieu agité et aéré. La cause en est uniquement le plus grand nombre de cocons pondus par les parents. L'agitation influence ces derniers, et non pas, ainsi qu'on pourrait le penser, les cocons pendant leur incubation.

A $20^{\circ}$ sembleraient se produire des phénomènes semblables. Mais la variabilité plus élevée des données fait qu'aucune différence n'atteint le seuil de signification de $95 \%$ généralement admis.

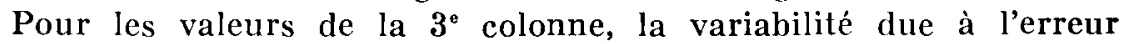
est même supérieure à celle qui est due à l'influence de l'aérateur! Ceite influence sur la reproduction à $20^{\circ}$ n'est donc pas démontrée comme elle l'est à $15^{\circ}$.

Il se pourrait, en définitive, qu'à certaines températures au moins, l'estimation du taux de reproduction fournie au paragraphe suivant soit un peu faible par rapport à ce que serait ce laux en eau courante. Mais ceci ne peut, a priori, rien enlever à la valeur de la comparaison entre les trois espèces rhéophiles, traitées de façon identique en eau stagnante.

\section{3. - Le taux de reproduction}

Les résultats figurent au tableau II, qui donne la valeur des mêmes paramètres pour $D$. gonocephala que la publication précédente [PATteE 1969a] pour Crenobia alpina el Polycelis felina. L'influence de la température apparaît de façon très précise.

Aucune ponte n'a lieu dans les lots élevés à $5^{\circ}$, bien que certains de ceux-ci soient actuellement âgés de 3 ans. D'autres individus, qui produisaient des cocons fertiles à $15^{\circ}$, ont cessé de pondre depuis leur transfert à $5^{\circ}$ en février 1969. La stérilité causée par le froid est donc absolue, que les organes génitaux soient préalablement développés ou non. Ce résultat diffère de celui obtenu par ABELoos [1930] qui recueillait des pontes à toutes les températures comprises entre 5 et $20^{\circ}$, mais correspond davantage aux observations de Steinmann [1907] et de Stankovic [1935] dans la nature.

Pas plus que la ponte des adultes, le développement des cocons n'est possible à $5^{\circ}$. Ainsi, des cocons pondus à $15^{\circ}$ par un lot 


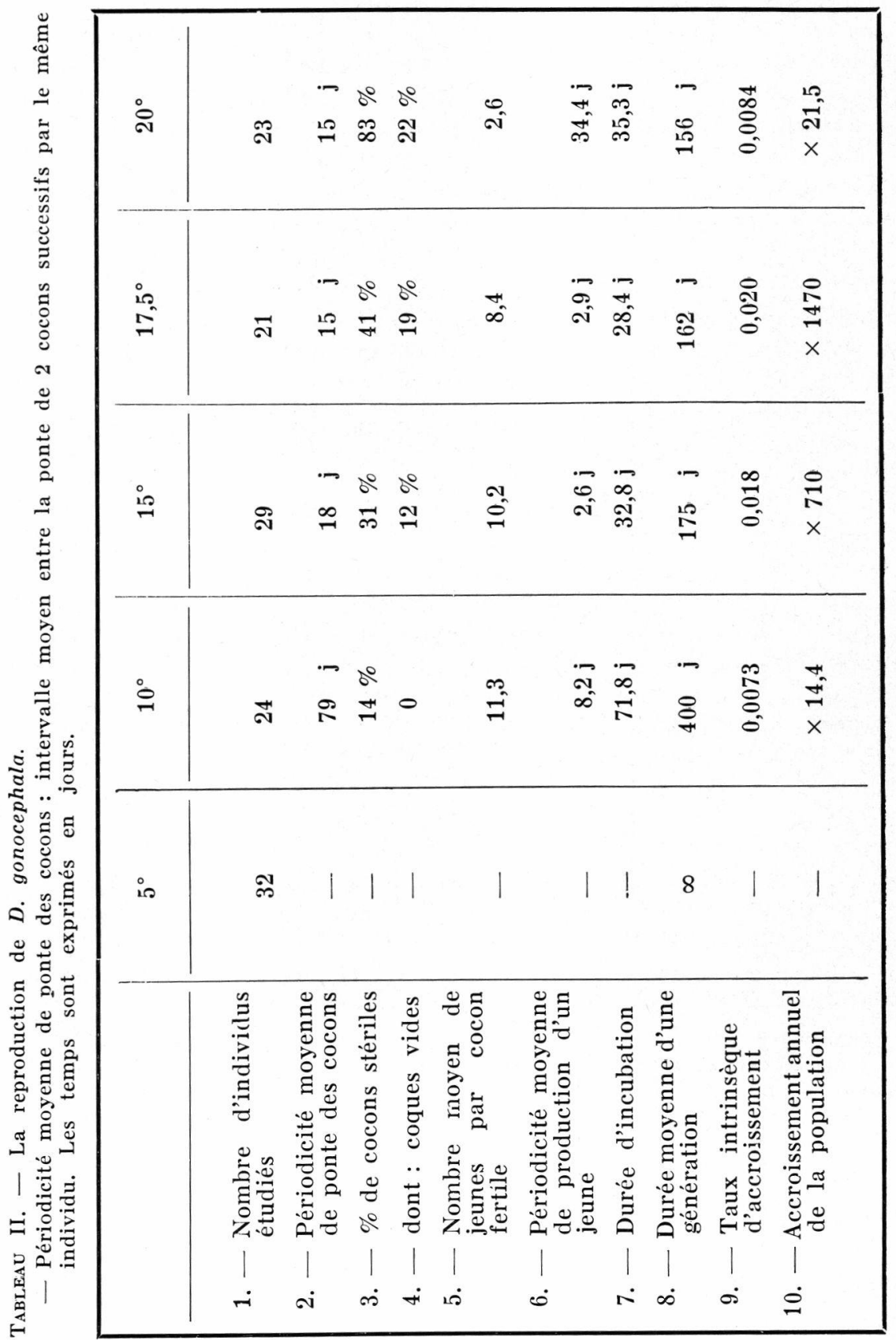


de parents ont été divisés en 2 groupes. L'un, servant de témoin à $15^{\circ}$, s'est révélé fertile. L'autre $(15$ cocons $)$, transféré à $5^{\circ}$, n'a donné naissance à aucun jeune.

La ponte a lieu de 10 à $20^{\circ}$. Elle est de nouveau nulle à $22,5^{\circ}$, qui fait partie de la zone létale [Pattee 1968]. La ligne $2 \mathrm{du}$ tableau montre qu'elle s'accélère à partir de $10^{\circ}$ pour atteindre un maximum vers 17,5 et $20^{\circ}$. La proportion de cocons stériles, faible à $10^{\circ}$, augmente progressivement jusqu'à atteindre la valeur élevée de $83 \%$ à $20^{\circ}$ (ligne 3 ); bien que le nombre de cocons produits à cette température soit encore important, peu d'entre cux donnent naissance à des nouveaux-nés. Les coques vides précédemment mentionnées s'observent à partir de $15^{\circ}$ (ligne 4 ). La température modifie également le nombre d'œufs qui se développent dans chaque cocon : la ligne 5 montre que ce nombre est plus important au froid qu'à la chaleur. Ces diverses actions, jouant en sens inverse, se traduisent, en définitive, par la production nette d'un certain nombre de jeunes, pour laquelle la ligne 6 fait apparaître un optimum aux environs de 15 et $17,5^{\circ}$. A $20^{\circ}$, la faiblesse de cette production, qui est inférieure même à celle relevée pour $10^{\circ}$, s'explique par la fertilité limitée des cocons et des oufs qu'ils renferment.

Après être passée par un minimum à $17,5^{\circ}$, la durée d'incubation (ligne 7 ) augmente de nouveau à $20^{\circ}$. Il semble, en fait, qu'à cette température, les jeunes éprouvent des difficultés à éclore. Leur développement proprement dit s'achève assez rapidement, et on peut ensuite, sous un éclairage intense, les voir se déplacer à l'intérieur du cocon. Lorsqu'ils éclosent enfin, ils sont souvent pigmentés de noir, signe de leur âge avancé.

La durée d'une génération (ligne 8) est mesurée comme le lemps que met un nouveau-né à produire un autre nouveau-né. Elle inclut donc la durée d'incubation. A $10^{\circ}$, elle est extrêmement longue, et atteint 400 jours. Pour Crenobia alpina, la valeur correspondante était de 378 jours, chiffre assez voisin. La comparaison est intéressante, car elle permet de répondre à la question : la taille 2 fois plus importante de $D$. gonocephala peut-clle constituer un handicap pour l'espèce en allongeant sa durée de développement, en retardant sa maturité sexuelle et en réduisant, de ce fait, sa capacité de reproduction? Il semble que non, lorsque la nourriture disponible est en quantité suffisante.

La ligne 8 du tableau montre en outre que la durée d'une génération diminue constamment lorsque la température s'élève. Dans ce cas particulier, $20^{\circ}$ représentent l'optimum. Abeloos [1930] compte une duréc de 5 à 6 mois entre l'éclosion d'un individu et sa première ponte. La valeur correspondante est ici d'environ 5 mois à $15^{\circ}$. 
Taux de ponte et durée d'une génération se combinent pour déterminer le taux intrinsèque d'accroissement naturel d'une population, représenté par la lettre $r$ [Pattee 1969a]. C'est là le seul coefficient permettant une comparaison interspécifique du rendement global de la reproduction. Les valeurs de $r$ figurent à la ligne $9 \mathrm{du}$ tableau. L'optimum de la reproduction se situe aux environs de 15 à $17,5^{\circ}$, concordant avec la "température sexuelle » notée par Steinmann [1907]. Le rendement est nettement plus faible de part et d'autre de cet optimum, pour les raisons que nous avons précédemment analysées et qui sont différentes dans les deux cas (lenteur des processus à $10^{\circ}$, fertilité réduite à $20^{\circ}$ ).

La $10^{\circ}$ ligne donne enfin un exemple plus concret d'application du coefficient $r$ : le calcul de l'effectif théorique atteint, après un an, par une population composée à l'origine d'un seul individu, autrement dit, le nombre par lequel se trouverait multiplié, chaque année, l'effectif de la population si elle était constamment maintenue dans les conditions de l'élevage.

\section{4. - Discussion}

La figure 1 permet de comparer, pour les trois espèces, les valeurs du taux intrinsèque d'accroissement, suivant la température. Il est intéressant de considérer les zones dans lesquelles le taux de l'une des Planaires est supérieur à celui de ses voisines. Dans les conditions standard de l'expérience, $C$. alpiza l'emporte jusqu'à $8^{\circ}$ environ, $P$. felina de 8 à $15^{\circ}$ environ et $D$. gonocephala au-dessus de $15^{\circ}$.

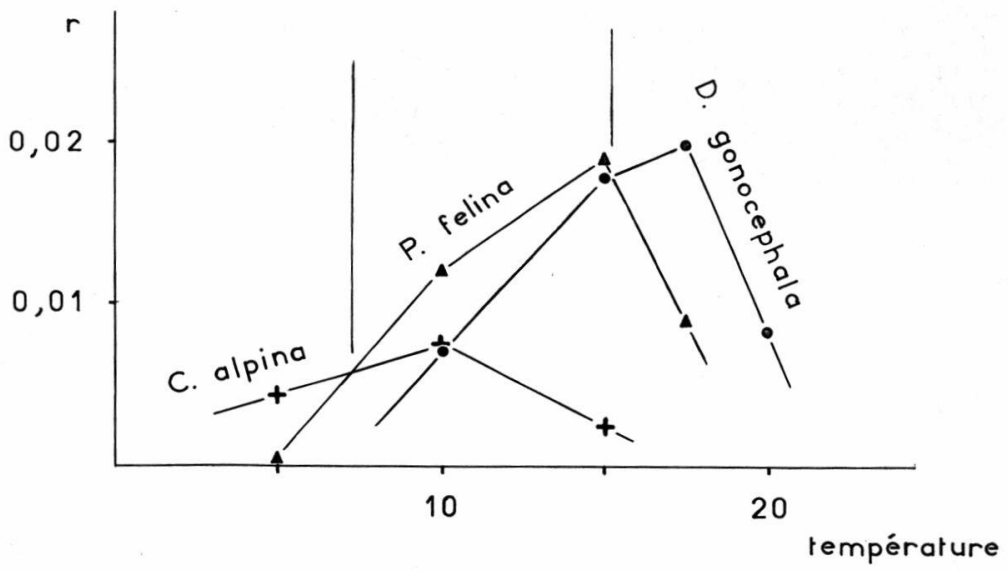

Fig. 1. - Variation du taux intrinsèque d'accroissement $r$ en fonction de la température chez les trois espèces de Planaires rhéophiles. Les tracés concernant $C$. alpina et $P$. felina sont reportés de Pattee [1969 a]. 
Or le raisonnement tend à démontrer que, dans la mesure où plusieurs espèces cherchent à exploiter les mêmes ressources du milieu, l'issue de la compétition qu'elles se livrent alors dépend de leur capacité reproductrice [Hardin 1960, PatTeE 1969a]. De fait, la succession des zones naturelles se trouve reflétée dans la figure 1, et même de façon assez précise : BEAUChaMP et Ullyotr [1932] d'une part, Alause [1968] d'autre part, donnent justement la valeur de $15^{\circ}$ comme limite inférieure de $D$. gonocephala lorsque celle-ci est en contact avec $P$. felina. L'hypothèse qui nous a servi de point de départ se trouve donc vérifiée. Dans les cas considérés, le mécanisme qui règle l'étagement peut se concevoir ainsi : l'animal se montre adapté physiologiquement à une zone thermique limitée, à l'intérieur de laquelle sa spécialisation lui permet un meilleur rendement reproductif. C'est grâce à ce dernier qu'il élimine ses rivaux par compétition.

Dans le cas de nos Planaires, il faut s'attendre à ce que la situation soit compliquée, sur le terrain, par l'intervention d'autres facteurs, le courant en premier lieu : bien que $P$. felina soit aussi un animal rhéophile, ses capacités de résistance à l'entrainement sont plus réduites que celles de ses rivales [Dorier et Vaillant 1954, Pattee et Bournaud 1970]. Il est logique, dans ces conditions, qu'en eau agitée, les secondes tendent à empiéter sur le domaine thermique de la première. Un tel phénomène a été mis en évidence aux points de rencontre entre $C$. alpina et $P$. felina [PATtee 1969b]. Entre $P$. felina et $D$. gonocephala, il jouerait dans le sens d'un déplacement de la limite de $15^{\circ}$ vers des températures plus basses.

A l'extrême, Becker [1960] signale des régions entières d'où $P$. felina est absente, pour la raison précédente ou pour une autre. $C$. alpina et $D$. gonocephala se trouvent alors en présence. Selon notre schéma théorique, les tracés correspondant à leurs capacités reproductrices se croisent vers $10^{\circ}$. Il serait intéressant de savoir si la limite de leurs deux zones naturelles coïncide avec cette température. BEcker donne, nous l'avons vu, pour l'écologie de $D$. gonocephala, des niveaux thermiques plus bas que ceux des autres auteurs. L'absence fréquente de $P$. felina n'en seraitelle pas la cause?

A la lumière de ces résultats, que doit-on penser de l'eurythermie de D. gonocephala, si apparente sur le terrain? S'agit-il, selon la phrase d'Alause [1968], d'une notion « à prendre au musée des horreurs? $\gg$ Il convient, en fait, de dissocier 2 phénomènes : celui de tolérance et celui de capacité reproductive. En ce qui concerne le premier, toutes ces Planaires survivent indéfiniment aux températures basses et voisines de $0^{\circ}$, telles qu'elles règnent en hiver dans nos ruisseaux. A partir de cette origine commune, les zones des trois espèces, de $C$. alpina à $D$. gonoce- 
phala, s'étendent de plus en plus loin vers les températures éle. vées. De ce point de vue, il y a donc eurythermie croissante.

Du point de vue de l'activité physiologique, il n'en est pas de même. Les longueurs d'abscisses couvertes par les trois tracés de la figure 1 ne diffèrent pas sensiblement entre elles : chaque espèce devient féconde, puis perd de nouveau sa fécondité à ur : température légèrement plus élevée que la précédente. Cette specialisation, mentionnée précédemment, fait donc apparaître entre elles, non plus une différence d'eurythermie, mais une différence de thermophilie. Etant donné le rôle que semble jouer la capacité reproductive de ces Planaires dans leur répartition naturelle, c'est cette notion de thermophilie croissante de l'une à l'autre qui caractérise le mieux leur écologie. Dans les ruisseaux, les zones à température moyenne relativement élevée que réclame $D$. gonocephala sont généralement des zones éloignées de la source, chaudes en été et froides en hiver. Et c'est seulement aux particularités de ces variations thermiques qu'est due l'apparente eurythermie de l'animal dans la nature.

\section{RÉSUME}

D. gonocephala se présente comme un animal relativement thermophile, doué d'une capacité reproductrice supérieure à celle de $C$. alpina au-dessus de $10^{\circ}$, supérieure à celle de $P$. felina au-dessus de $15^{\circ}$. Une telle capacité explique l'élimination des deux dernières espèces par la première dans les zones les plus chaudes de nos ruisseaux.

\section{THE TEMPERATURE RELATIONS OF SOME FRESH-WATER PLANARIANS AND THEIR INCIDENCE ON ECOLOGY. 4. - THE REPRODUCTION OF DUGESIA GONOCEPHALA.}

D. gonocephala appears to be a relatively thermophilous Triclad. Its reproductive capacity is greater than that of Crenobia alpina above $10^{\circ} \mathrm{C}$ and greater than that of Polycelis felina above $15^{\circ} \mathrm{C}$. This greater reproductive capacity explains the competitive replacement of the two latter species by $D$. gonocephala in the warmer zones of streams.

\section{DER TEMPERATURFAKTOR UND DIE OEKOLOGIE EINIGER SUESSWASSERPLANARLEN. 4. - DIE REPRODUKTION VON DUGESIA GONOCEPHALA.}

D. gonocephala ist offenbar ein relativ thermophiles Tier. Es hat bei einer Temperatur von über $10^{\circ} \mathrm{C}$ eine höhere Vermehrungsfähigkeit als Crenobia alpina und bei einer von über $15^{\circ} \mathrm{C}$ eine höhere als Polycelis felina. Damit wird das Eindringen von D. gonocephala in die wärmeren Abschnitte unserer Bäche auf Kosten dieser beiden Arten erklärt. 


\section{TRAVAOX CITÉS}

Abeloos (M.). 1929. - Les facteurs déterminant la ponte des cocons chez Planaria gonocephala Dugès. Bull. Soc. zool. Fr., 54: 291296.

Abeloos (M.). 1930. - Recherches expérimentales sur la croissance et la régénération chez les Planaires. Bull. biol., 64: 1-140.

Alause (P.). 1968. - Contribution à l'écologie des Planaires d'eaux courantes: Observations en Languedoc méditerranéen. Le facteur acidité carbonique. Thèse, Montpellier. $730 \mathrm{p}$.

Beauchamp (R.S. A.) et UllyotT (P.). 1932. - Competitive relationships between certain species of fresh-water Triclads. J. Ecol., 20: $200-208$.

BECKER (E.). 1960. - Zur geographischen Verbreitung und ökologic der tricladen Turbellarien in Württemberg. Jh. Ver. vaterl. Naturk. Württ., 115: 267-305.

BlNazzI (M.). 1955. - Appunti sulla distribuzione dei Tricladi in Italia. Boll. Zool, 22: 149-164.

Benazzi (M.). 1960. - Evoluzione cromosomica e differenziamento razziale e specifico nei Tricladi. Acc. Naz. Lincei. 47: 273-297.

BläsıNg (I.). 1953. - Experimentelle Untersuchungen über den Umfang der ökologischen und physiologischen Toleranz von Planuria alpina Dana und Planaria gonocephala Dugès. Zool. Jb., Abt. allg. Zool. Physiol., 64: 112-152.

DAнм (A. G.). 1958. - Taxonomy and ecology of five species groups in the family Planariidae (Turbellaria Tricladida Paludicola). Malmö, $241 \mathrm{p}$.

Dorier (A.) et Vaillant (F.). 1954. - Observations et expériences relatives à la résistance au courant de divers invertébrés aquatiques. Trav. Lab. Hydrobiol. Grenoble, 45-46: 9-31.

Flössner (D.). 1963. - Zur ökologie der Bergbachtrikladen im Erzgebirge. Limnologica, Berlin, 1 : 35-44.

Hardin (G.). 1960. - The competitive exclusion principle. Science, 131: $1292-1297$.

VAN OYE (E. L.). 1941. - Verbreitung und ökologie der paludicolen Tricladen in Belgien. Arch. Hydrobiol., 38: 110-147.

Pattee (E.). 1968. - Coefficients thermiques et écologie de quelques Planaires d'eau douce. 2. Tolérance de Dugesia gonocephala. Annls Limnol., 4: 99-104.

Pattee (E.). 1969 a. - Coefficients thermiques et écologie de quelques Planaires d'eau douce. 3 . La reproduction des deux espèces montagnardes. Annls Limnol., 5: 9-24.

Pattee (E.). $1969 \mathrm{~b}$. - Contribution expérimentale à l'écologie de la Planaire alpine, Crenobia alpina (Dana). Bull. Soc. zool. Fr., 94: 269-276.

Pattee (E.) et Bournaud (M.). 1970. - Etude expérimentale de la rhéophilie chez des Planaires Triclades d'eau courante. Schw.Z. Hydrol. 32: 181-191.

Stankovic (S.). 1935. - Uber die Verbreitung und ökologie der Quelltricladen auf der Balkanhalbinsel. Zoogeographica, 2: 147203. 
Steinmann (P.). 1907. - Geographisches und Biologisches von Gebirgsbachplanarien. Arch. Hydrobiol., 2: 186-217.

Vojgt (W.). 1895. - Planaria gonocephala als Eindringling in das Verbreitungsgebiet von Planaria alpina und Polycelis cornuta. Zool. Jb., Abt. Syst. ökol. Geogr., 8: 131-176.

Section de Biologie Animale et Zoologie, Faculté des Sciences, Lyon. 